\title{
Near-IR spectroscopy of OH/IR stars in the Galactic centre ${ }^{\star}$
}

\author{
E. Vanhollebeke ${ }^{1}$, J. A. D. L. Blommaert ${ }^{1}$, M. Schultheis ${ }^{2}$, B. Aringer ${ }^{3}$, and A. Lançon ${ }^{4}$ \\ 1 Instituut voor Sterrenkunde, K.U.Leuven, Celestijnenlaan 200B, 3001 Leuven, Belgium \\ e-mail: evelien@ster.kuleuven.be \\ 2 CNRS UMR6091, Observatoire de Besançon, BP 1615, 25010 Besançon, France \\ 3 Institut für Astronomie, Türkenschanzstrasse 17, 1180 Wien, Austria \\ ${ }^{4}$ Observatoire Astronomique, 11 rue de l'Université, 67000 Strasbourg, France
}

Received 13 March 2006 / Accepted 18 April 2006

\begin{abstract}
Context. Based on the expansion velocities of their circumstellar shells, Galactic centre (GC) OH/IR stars can be divided into two groups that are kinematically different and therefore believed to have evolved from different stellar populations.

Aims. We studied the metallicity distribution of the $\mathrm{OH} / \mathrm{IR}$ stars population in the GC on the basis of a theoretical relation between $E W(\mathrm{Na}), E W(\mathrm{Ca})$, and $E W(\mathrm{CO})$ and the metallicity.

Methods. For $70 \mathrm{OH} / \mathrm{IR}$ stars in the GC, we obtained near-IR spectra. The equivalent line-widths of Na I, Ca I, ${ }^{12} \mathrm{CO}(2,0)$, and the curvature of the spectrum around $1.6 \mu \mathrm{m}$ due to water absorption were determined.

Results. The near-IR spectrum of OH/IR stars is influenced by several physical processes. OH/IR stars are variable stars suffering high mass-loss rates. The dust that is formed around the stars strongly influences the near-IR spectra and reduces the equivalent linewidths of $\mathrm{Na}$ I and Ca I. A similar effect is caused by the water content in the outer atmosphere of the OH/IR star. Because of these effects, it is not possible to determine the metallicities of these stars with our low-resolution near-infrared spectroscopy.
\end{abstract}

Key words. stars: AGB and post-AGB - stars: late-type - stars: mass-loss - Galaxy: bulge - Galaxy: center - infrared: stars

\section{Introduction}

The inner region of our Galaxy has been extensively searched for $\mathrm{OH} / \mathrm{IR}$ stars in the past two decades. The studies of the GC region are severly hampered by extinction and also source confusion, especially in the visible wavelength range. Searches for $\mathrm{OH} / \mathrm{IR}$ stars at radio wavelengths at the $1612 \mathrm{MHz} \mathrm{OH}$ maser line have therefore been very useful and also provided kinematic information on the detected stars. Lindqvist et al. (1992b) searched for $\mathrm{OH} / \mathrm{IR}$ stars in six VLA primary beam fields and identified $134 \mathrm{OH} / \mathrm{IR}$ stars. Further searches, also using ATCA, were conducted by Sevenster et al. (1997), who found 145 new $\mathrm{OH}$ masering sources in the Bulge, and Sjouwerman et al. (1998), who discovered an additional 52 new OH/IR stars in the GC region. A review of Asymptotic Giant Branch (AGB) stars in the GC region can be found in Habing \& Whitelock (2003).

Lindqvist et al. (1992a) studied the spatial and kinematic properties of their $\mathrm{OH} / \mathrm{IR}$ sample and divided the stars into two groups on the basis of their outflow velocities. The OH/IR stars with low expansion velocities $\left(v_{\exp }<18.0 \mathrm{~km} \mathrm{~s}^{-1}\right)$ have a larger spread in latitude and a larger velocity dispersion with respect to the Galactic rotation than the group with higher expansion velocities. This was also found by Baud et al. (1981) for OH/IR stars in the Galactic disk. The low-expansion velocity stars are expected to be older objects with larger peculiar motions, whereas the other group may have a different formation history; it might be a later addition to the GC, possibly via a merger. The outflow velocity of the circumstellar shell is related to the luminosity of the star and to the properties of the dust in the circumstellar shell (Habing et al. 1994; Elitzur et al. 2003).

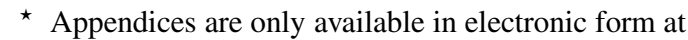
http://wWw .edpsciences.org
Several groups have been searching for the infrared counterparts of the OH maser sources (Jones et al. 1994; Blommaert et al. 1998; Wood et al. 1998; Ortiz et al. 2002). It would be expected that the "older" stars have lower luminosities than the high-expansion group. Blommaert et al. (1998) found that the high expansion velocities group contains higher luminosity stars but that there was also a large overlap in the luminosity distributions of the 2 groups. Ortiz et al. (2002) also compared the measured luminosities using a larger number of stars and did not find any evidence of a distinction on the basis of luminosities between the 2 groups. The disadvantage of the latter study is that the luminosities were not corrected for variability and that the luminosities spread over a wider range. Nevertheless, it seems that the differences in luminosities between the 2 groups, if they exist, are not very big. If the luminosity is not sufficient for explaining the differences in expansion velocities then it is expected that differences in the gas-to-dust ratios and thus metallicities of the stars must exist. It was demonstrated by Wood et al. (1992) for $\mathrm{OH} / \mathrm{IR}$ stars in the Large Magellanic Cloud and by Blommaert et al. (1993) for OH/IR stars in the outer Galaxy that the expansion velocities are low, even though the $\mathrm{OH} / \mathrm{IR}$ stars have high luminosities. In both the LMC and in the outer Galaxy, it is expected that the stars indeed have low metallicities. The next logical step would be to investigate the metallicities of GC OH/IR stars.

Schultheis et al. (2003) obtained near-IR spectra of 107 sources with mid-infrared excess selected from the ISOGAL survey (Omont et al. 2003), including $15 \mathrm{OH} / \mathrm{IR}$ sources. With an empirical formula based on the near-infrared spectroscopy of K and M giants (Ramírez et al. 2000; Frogel et al. 2001), Schultheis et al. (2003) tentatively estimated $[\mathrm{Fe} / \mathrm{H}]$ for all of the ISOGAL sources. Although Schultheis et al. (2003) indicate that the Ramírez et al. (2000) formula does not actually 
measure metallicity for an individual spectrum of a strongly variable star, it was found that it might still be used to find an average metallicity.

In this paper we discuss our attempt to apply the results of Schultheis et al. (2003) on medium-resolution near-IR spectra on a sample of GC OH/IR stars. We take a look at the influence of the water content on the atomic lines $\mathrm{Na}$ I and $\mathrm{Ca}$ I, discuss how periodicity can influence the water content and therefore also the near-IR spectrum, and we study a grid of dust models and show that hot dust also has an influence on the near-IR spectrum.

The sample, observations, and data-reduction are described in the next section. In Sect. 3, we explain our method of analysis and compare spectra we have in common with other authors. The problems one encounters analysing near-IR spectra of $\mathrm{OH} / \mathrm{IR}$ stars will be discussed in Sect. 4. Finally in Sect. 5 we summarise and come to the conclusions.

\section{Observations and data reduction}

The sample consists of $70 \mathrm{OH} / \mathrm{IR}$ stars located in the GC region. Almost all stars were selected from Lindqvist et al. (1992b) and Sjouwerman et al. (1998). Our sample also includes the 15 $\mathrm{OH} / \mathrm{IR}$ stars observed by Schultheis et al. (2003) so that we can compare our results and investigate the effect of the variability of this type of stars. We also selected the three "highvelocity" $\mathrm{OH} / \mathrm{IR}$ stars that were detected in the direction of the GC (van Langevelde et al. 1992). As we wanted to apply the metallicity versus $E W(\mathrm{Na}), E W(\mathrm{Ca})$, and $E W(\mathrm{CO})$ calibration used in Ramírez et al. (2000) and Frogel et al. (2001) to our sample, we also observed 11 stars from Ramírez et al. (1997, $K$ band spectra of 43 luminosity class III stars from K0 to M6) and Ramírez et al. (2000, $K$-band spectra of more than $110 \mathrm{M}$ giants in the Galactic bulge (GB)). Finally, to investigate the effects of the variability of our long period variable stars (LPVs) on our analysis, we measured variable stars from Lançon \& Wood (2000, spectra of cool, mostly variable, giant, and supergiant stars) (see Tables A.1-Table A.3).

The near-IR spectra were obtained with the $3.58 \mathrm{~m}$ NTT (ESO) at la Silla, Chile between June 28-30, 2003 using the red grism of the SOFI spectrograph. This resulted in spectra from $1.53 \mu \mathrm{m}$ up to $2.52 \mu \mathrm{m}$. Before the actual spectrum was obtained, the instrument was used in imaging mode to acquire the star in the slit. Several spectra were taken with the star in different places along the slit. Standard stars of spectral types $\mathrm{O}$ to $\mathrm{G}$ were observed as close as possible to the object stars' airmass in order to correct for telluric absorption features.

The data was reduced using the ESO-Munich Image Data Analysis System (ESO-MIDAS). The images were first corrected for cosmic ray hits. Several spectra of the same target along the slit were obtained and subtracted from each other to correct for the sky level. The images were flat-fielded using dome flats. A Gaussian fit perpendicular to the dispersion direction was used to subtract a one-dimensional spectrum out of the two-dimensional image: columns that fall within the FWHM of the Gaussian fit were added to the spectrum (3-4 columns on average). A correction for distortion along the slit was unnecessary. During the extraction process, a correction for bad pixels was made, they were left out of the spectrum, and no averaging was done around these pixels. The wavelength calibration was based on the spectrum of a xenon lamp. This image was also flat-fielded using the dome flats and corrected for bad pixels in the same way as the other spectra. The wavelength calibration resulted in a dispersion of typically $10.13 \AA /$ pixel and $\lambda / \Delta \lambda \approx 1000$.
Table 1. Definition of band passes for continuum and features (Schultheis et al. 2003).

\begin{tabular}{lc}
\hline \hline Feature & Band passes $[\mu \mathrm{m}]$ \\
\hline $\mathrm{Na}$ I feature & $2.204-2.211$ \\
Na I continuum \#1 & $2.191-2.197$ \\
Na I continuum \#2 & $2.213-2.217$ \\
Ca I feature & $2.258-2.269$ \\
$\mathrm{Ca}$ I continuum \#1 & $2.245-2.256$ \\
$\mathrm{Ca}$ I continuum \#2 & $2.270-2.272$ \\
${ }^{12} \mathrm{CO}(2,0)$ feature band head & $2.289-2.302$ \\
${ }^{12} \mathrm{CO}(2,0)$ continuum \#1 & $2.252-2.258$ \\
${ }^{12} \mathrm{CO}(2,0)$ continuum \#2 & $2.284-2.291$ \\
$\mathrm{H}_{2} \mathrm{O}$ continuum & $1.629-1.720$ \\
$\mathrm{H}_{2} \mathrm{O}$ absorption wing 1 & $1.515-1.525$ \\
$\mathrm{H}_{2} \mathrm{O}$ absorption wing 2 & $1.770-1.780$ \\
\hline
\end{tabular}
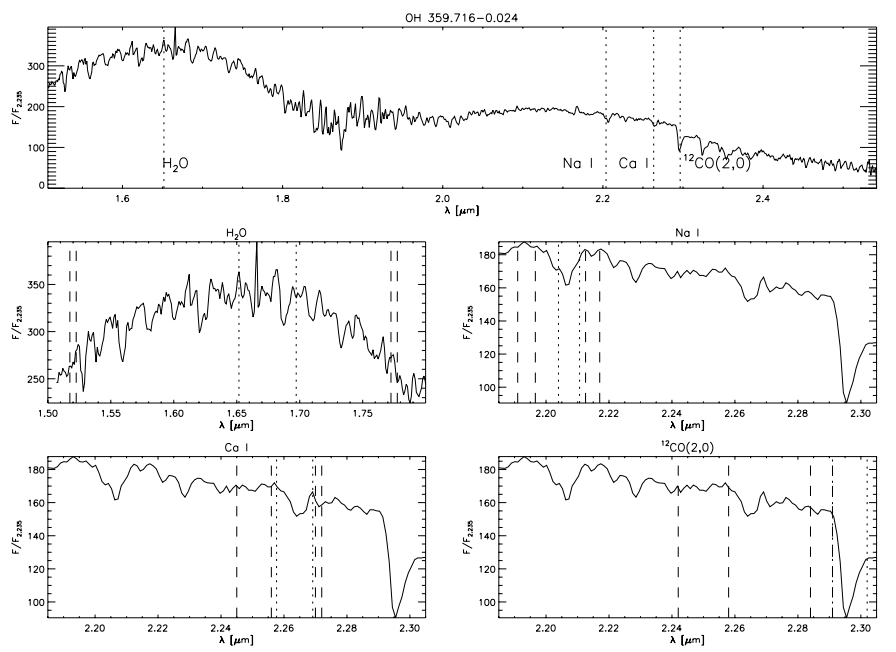

Fig. 1. An overview of the chosen definition of band passes for continuum and features. The top figure shows the overall spectrum for a typical OH/IR stars. The dotted lines indicate the central positions of the features. The other 4 figures show for each line the selected passband for the feature (dotted lines) and the selected passband for the continuum (dashed lines). (For the ${ }^{12} \mathrm{CO}(2,0)$ the feature's first dotted line overlaps with the last dashed line from the continuum.)

The standard stars were reduced in the same way. Thereafter they were divided by the Kurucz-model that corresponds to each one's spectral type. The resulting curve was used to correct the objects for telluric lines and also to correct for the instrumental response function. Different curves for different airmasses were made in order to correct for the airmass. The dereddening law used is based on Cardelli et al. (1989), $A_{\mathrm{v}}$ values came from Schultheis et al. (1999). The resulting spectra are shown in Figs. B.1 to B.8.

\section{Analysis}

\subsection{Equivalent line widths}

The equivalent line widths of $\mathrm{NaI}, \mathrm{Ca}$, and ${ }^{12} \mathrm{CO}(2,0)$ (see Tables A.1-A.3) were obtained in exactly the same way as by Schultheis et al. (2003, see also Ramírez et al. 1997; Lançon \& Wood 2000). They were measured relative to the selected continuum bands using the Image Reduction and Analysis Facility (IRAF) (see Table 1 and Fig. 1).

Ramírez et al. (1997) discuss how the equivalent-width measurements of $\mathrm{NaI}$ and $\mathrm{CaI}$ in medium-resolution spectra contain contributions from other elements such as $\mathrm{Sc}, \mathrm{Ti}, \mathrm{V}, \mathrm{Si}$, and 
S. Thus, it turns out that a significant contribution of the $\mathrm{NaI}$ and $\mathrm{Ca}$ I features is due to other species. In the studied spectral region (1.53-2.52 $\mu \mathrm{m})$, the $\mathrm{CN}$ molecule causes a noisy continuum (Origlia et al. 1993), introducing a pseudo-continuum opacity, which is clearly shown in the high-resolution spectrum of RX Boo (an M8 III star) in Wallace \& Hinkle (1996). According to Ramírez et al. (1997), the continuum bands that were used to determine the $E W(\mathrm{Ca})$ are affected by $\mathrm{CN}$ absorption for stars with $T_{\text {eff }}<3000 \mathrm{~K}$. However, synthetic CN spectra based on hydrostatic MARCS models for giant stars (for a description of such models see Aringer et al. (1997), in our case we assumed $\log \left(G\left[\mathrm{~cm} / \mathrm{s}^{2}\right]\right)=0.0$, solar mass, and elemental abundances) show that $\mathrm{CN}$ is important in all cool objects below $4000 \mathrm{~K}$, and even gets weaker below $3000 \mathrm{~K}$.

The effective temperature has a strong impact on the Ca I and $\mathrm{Na}$ I features: as $T_{\text {eff }}$ decreases, the equivalent widths of both features increase. Tables A.4, A.5, and A.6 give an overview of what the respective $\mathrm{Na}$ I, $\mathrm{Ca}$ I, and ${ }^{12} \mathrm{CO}(2,0)$ lines in a mediumresolution spectrum are really made of. The tables are based on the high-resolution spectrum of RX Boo in Wallace \& Hinkle (1996). For cooler oxygen-rich stars (Lançon \& Wood 2000), such as $\mathrm{OH} / \mathrm{IR}$ stars, $\mathrm{H}_{2} \mathrm{O}$ absorption, instead of $\mathrm{CN}$ absorption, becomes very important (see Sect. 4.3).

\subsection{Water absorption}

The amount of water was obtained by measuring the curvature in the spectrum around $1.6 \mu \mathrm{m}$, as in Schultheis et al. (2003). The apparent bump around $1.6 \mu \mathrm{m}$ is caused by strong and wide water-absorption bands around 1.4 and $1.9 \mu \mathrm{m}$. Formally, our measurements are equivalent widths of this bump relative to reference fluxes measured on either side of it in the wings of the water bands. As for the other equivalent line width measurements, its value is given in $\AA$, but it takes negative values when water absorption is present (see Tables A.1-A.3).

\subsection{Comparison with previous work}

We have 5 candidate Red Giant Branch (RGB) stars in common with Schultheis et al. (2003). These candidate RGB stars are probably genuine ones, since we see no significant difference between the Schultheis et al. (2003) observations of these stars and our own. Stars on the RGB are expected to show weak variations compared to $\mathrm{OH} / \mathrm{IR}$ stars and Miras.

Schultheis et al. (2003) observed the stars with the same instrument (SOFI), using the same grism. This results in spectra with the same resolution as our spectra. The time difference between these observations is about 3 years. The spectra (normalised at $2.235 \mu \mathrm{m}$ ) are compared in Fig. 2, and Table 2 gives the mean values and standard deviations for the stars we have in common. The absolute average differences in equivalent line widths for these RGB stars are consistent with scatter due to formal errors $(\sim 1 \AA)$. One expects these errors to come from data-reduction issues and changing of observing conditions during the night. The absolute average differences are: $0.25 \pm 0.17 \AA$ for $E W(\mathrm{Na}), 0.72 \pm 0.42 \AA$ for $E W(\mathrm{Ca})$, and $0.87 \pm 0.82 \AA$ for $E W(\mathrm{CO})$ (see also Fig. 3).

We have 8 late-type giants in common with Ramírez et al. (1997) and 6 red giants situated in the Bulge with Ramírez et al. (2000). A comparison was only made for $E W(\mathrm{Na})$ and $E W(\mathrm{Ca})$, since Ramírez et al. (1997) uses different continuum pass bands for obtaining $E W(\mathrm{CO})$. Tables 3 and 4 give the mean values for the compared equivalent line widths. The absolute average
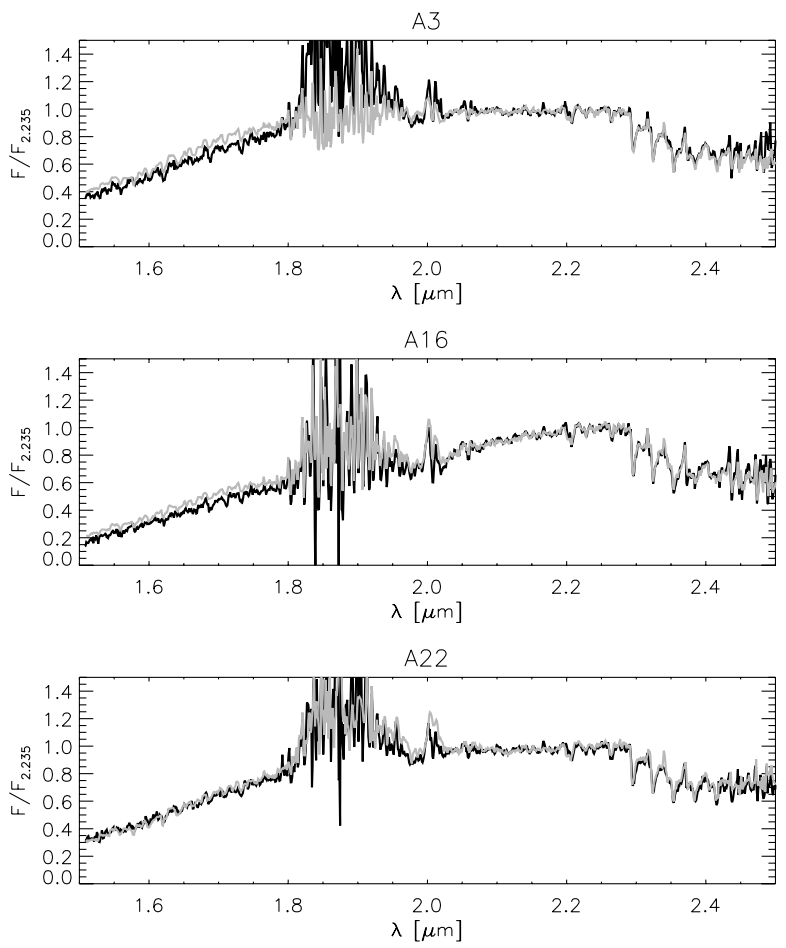

A45
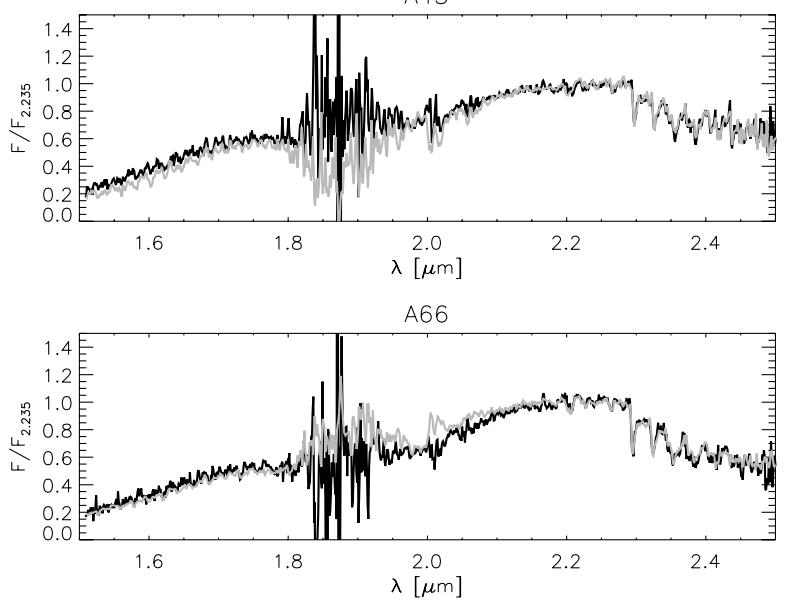

Fig. 2. RGB stars in common with Schultheis et al. (2003); black line: this work, gray line: Schultheis et al. (2003). The features between 1.8 and $1.9 \mu \mathrm{m}$ and around $2 \mu \mathrm{m}$ are due to the atmosphere.

differences with Ramírez et al. (1997) are: $0.39 \pm 0.13 \AA$ for $E W(\mathrm{Na})$ and $1.29 \pm 0.41 \AA$ for $E W(\mathrm{Ca})$. The absolute average differences with Ramírez et al. (2000) are $0.59 \pm 0.23 \AA$ for $E W(\mathrm{Na})$ and $0.66 \pm 0.35 \AA$ for $E W(\mathrm{Ca})$. The difference in $E W(\mathrm{Na})$ (Ramírez et al. 1997, 2000) and $E W(\mathrm{Ca})$ (Ramírez et al. $2000)$ is again consistent with scatter due to formal errors. There is no obvious reason why the difference for $E W(\mathrm{Ca})$ (Ramírez et al. 1997) is larger than $\sim 1 \AA$ (see also Fig. 4), although we have to keep in mind that there is a difference in resolution between our spectra and the spectra of Ramírez et al. (1997, 2000), which can influence the difference between the measured equivalent line widths. The data from Ramírez et al. (2000) can be downloaded from the internet. After rebinning the data to our lower resolution, the following values could be measured: $3.95 \pm 0.78$ for the $E W(\mathrm{Na})$ and $2.77 \pm 0.47$ for the $E W(\mathrm{Ca})$, which resembles the values we found better (see Table 3). A similar test for Ramírez et al. (1997) could not be done. 


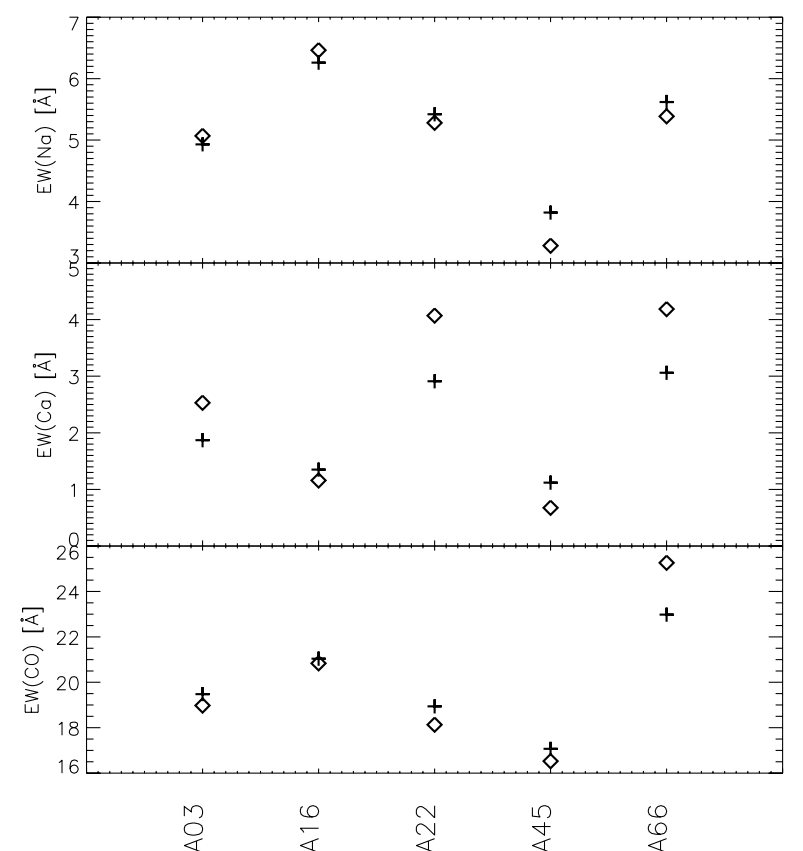

Fig. 3. Equivalent line width measurements for the candidate RGB stars in common with Schultheis et al. (2003). The plus-signs are taken from Schultheis et al. (2003), the diamonds from this work.

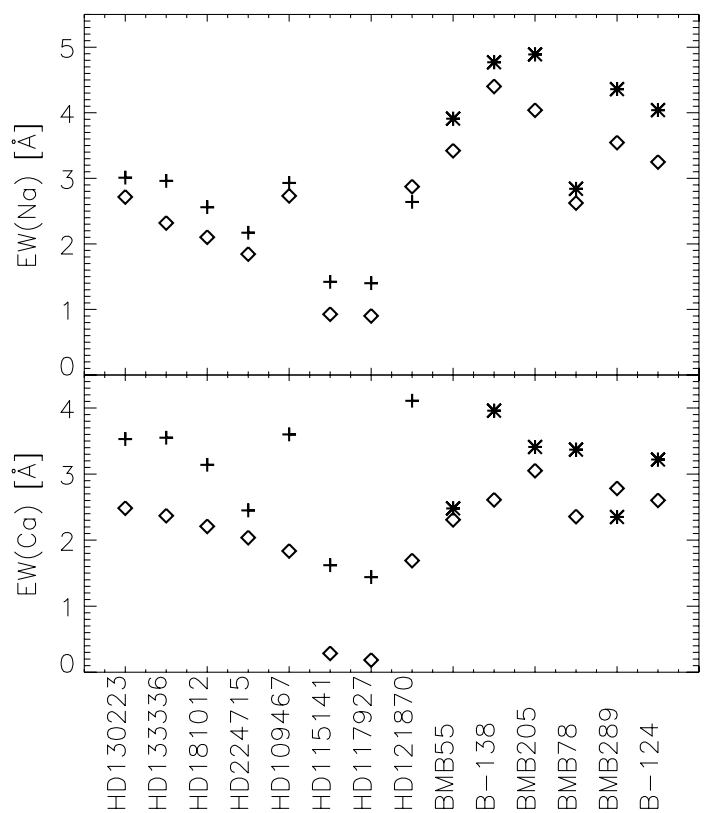

Fig. 4. Equivalent line width measurements for the stars in common with Ramírez et al. (1997) and Ramírez et al. (2000). The plus-signs are taken from Ramírez et al. (1997), the stars are from Ramírez et al. (2000) and the diamonds are from this work.

We have 7 luminous cool stars in common with Lançon \& Wood (2000). Three of these stars (WX Psc, AFGL 1686, and $\mathrm{U}$ Equ) are OH/IR stars (Lançon \& Wood 2000), which (not situated in the GC) are used as a comparison for the equivalent line width measurements. The 4 other stars have periods in the range of 120 to 270 days. Some of the stars were observed several times by Lançon \& Wood (2000). The differences in equivalent line widths between the measurements based on the available spectra of Lançon \& Wood (2000) and our measurements are shown in Fig. 5. The differences seen in this plot are large in comparison with the differences seen in Figs. 3 and 4, especially for $E W(\mathrm{CO})$, and can not be explained by formal errors

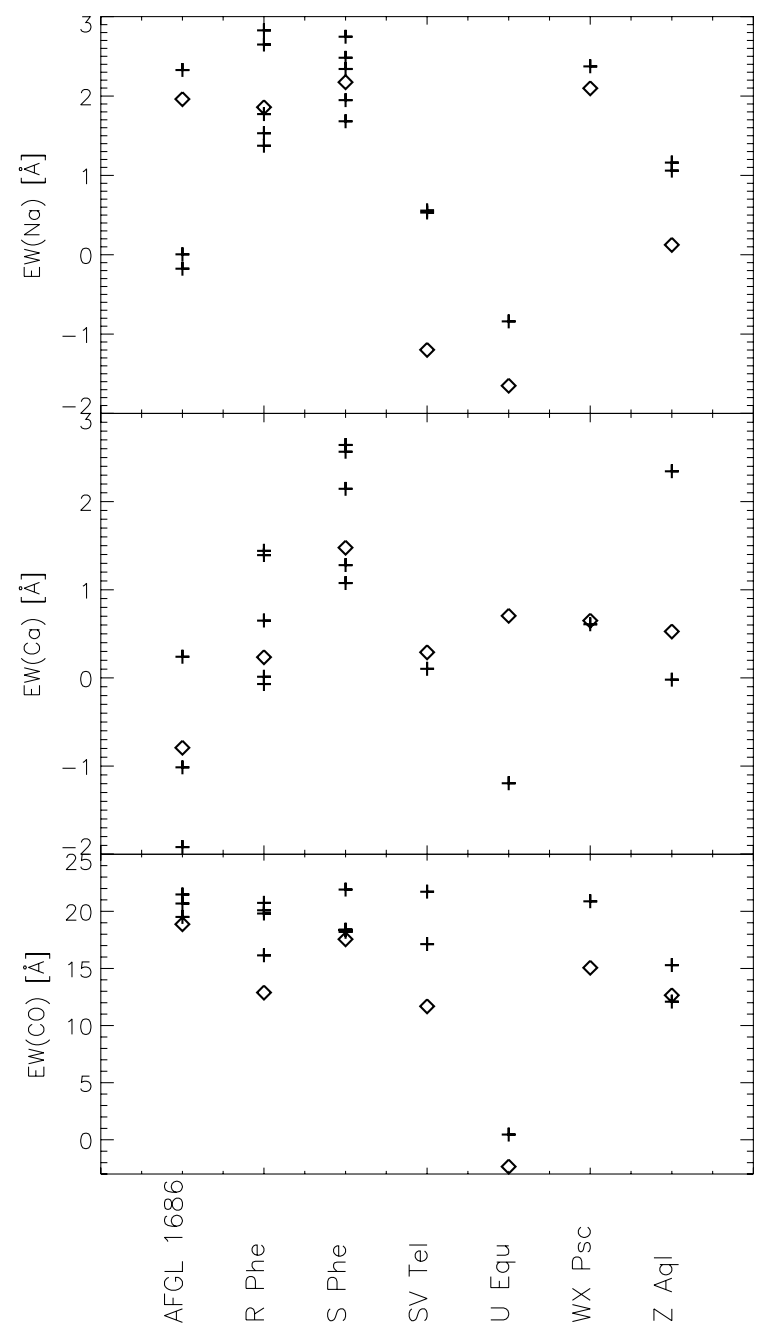

Fig. 5. Variations in the equivalent widths for stars in common with Lançon \& Wood (2000). The plus-signs indicate values taken from Lançon \& Wood (2000), the diamonds those from this work.

Table 2. Mean values for the equivalent line widths for the stars in common with Schultheis et al. (2003).

\begin{tabular}{ccc}
\hline \hline & this work & Schultheis et al. (2003) \\
\hline $\mathrm{Na} \mathrm{I}$ & $5.10 \pm 0.74$ & $5.21 \pm 0.67$ \\
$\mathrm{Ca}$ I & $2.52 \pm 1.29$ & $2.06 \pm 0.74$ \\
${ }^{12} \mathrm{CO}(2,0)$ & $19.95 \pm 2.48$ & $19.90 \pm 1.69$ \\
\hline
\end{tabular}

alone. The variability of the stars is responsible for the large variations in the equivalent line widths (see Sect. 4.4). Our measured equivalent line widths do fall within the range of equivalent line widths based on the spectra of Lançon \& Wood (2000, see Fig. 5). We can expect that the OH/IR stars that we have in common with Schultheis et al. (2003) will show similar variations in their equivalent line widths, as illustrated in Fig. 6. The mean absolute differences for the OH/IR stars in common with Schultheis et al. (2003) concerning the equivalent line widths are: $1.56 \pm 1.17 \AA$ for $E W(\mathrm{Na}), 1.14 \pm 1.16 \AA$ for $E W(\mathrm{Ca})$, $3.60 \pm 2.47 \AA$ for $E W(\mathrm{CO})$ and $171.81 \pm 193.03 \AA$ for the water absorption. These variations are caused by the variability of these $\mathrm{OH} / \mathrm{IR}$ stars, as will be explained in the following section.

Figure 7 gives an overview of the equivalent line widths for $\mathrm{Na}$ I, Ca I, and ${ }^{12} \mathrm{CO}(2,0)$ (see Sect. 3.1 and Table 1). The fourth histogram also gives the amount of water. Only the OH/IR stars are considered in this figure. Notice that the $E W(\mathrm{Na}), E W(\mathrm{Ca})$, 


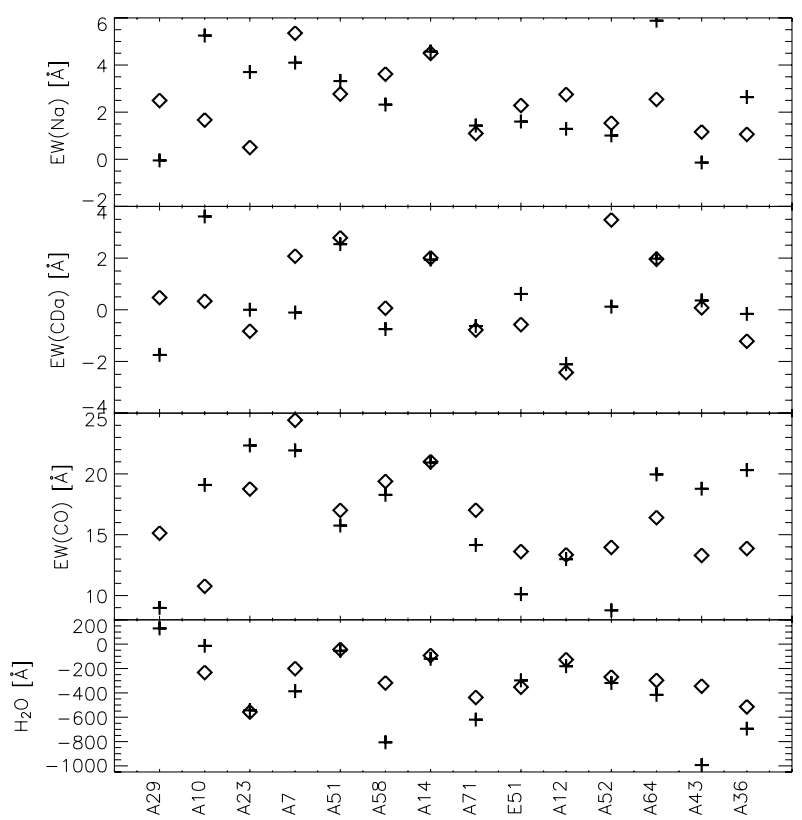

Fig. 6. Equivalent line-width measurements for the OH/IR stars in common with Schultheis et al. (2003). The plus-signs indicate values taken from Schultheis et al. (2003), the diamonds those from this work.

Table 3. Mean values for the equivalent line widths for the stars in common with Ramírez et al. (1997).

\begin{tabular}{lcc}
\hline \hline & this work & Ramírez et al. (1997) \\
\hline $\mathrm{Na}$ I & $2.05 \pm 0.62$ & $2.39 \pm 0.54$ \\
$\mathrm{Ca}$ I & $1.64 \pm 0.70$ & $2.93 \pm 0.82$ \\
\hline
\end{tabular}

Table 4. Mean values for the equivalent line widths for the stars in common with Ramírez et al. (2000).

\begin{tabular}{lccc}
\hline \hline & this work & Ramírez et al. (2000) & $\begin{array}{c}\text { Ramírez et al. (2000) } \\
\text { after rebinning }\end{array}$ \\
\hline $\mathrm{Na}$ I & $3.55 \pm 0.45$ & $4.13 \pm 0.54$ & $3.95 \pm 0.78$ \\
$\mathrm{Ca}$ I & $2.62 \pm 0.20$ & $3.13 \pm 0.48$ & $2.77 \pm 0.47$ \\
\hline
\end{tabular}

$E W(\mathrm{CO})$, and the $E W\left(\mathrm{H}_{2} \mathrm{O}\right)$ are all in the same unit $\AA$. The $E W\left(\mathrm{H}_{2} \mathrm{O}\right)$ are all negative values, indicating water absorption in the spectrum around $1.6 \mu \mathrm{m}$, as can be seen from the spectra of the $\mathrm{OH} / \mathrm{IR}$ stars in Fig. B.1. A negative value for $E W(\mathrm{Ca})$ and $E W(\mathrm{Na})$ indicates that the line could not been measured, since we do not expect these lines in emission. Figure 8 gives an example of this. It is especially clear for Ca I why the feature could not be measured: the second continuum band pass shows a very deep feature, which causes the flux measurement in the feature to be higher than in the continuum. This is also the case for the $\mathrm{Na}$ I feature, but here the first continuum band pass causes it (less clear than for the $\mathrm{Ca}$ I feature).

Figure 2 in Ramírez et al. (2000) gives a 1-5 ̊ range for $E W(\mathrm{Ca})$ and a 2-6 $\AA$ range for $E W(\mathrm{Na})$ for a sample of red giants in the GB. Figure 7 shows that our ranges for the equivalent widths for both $\mathrm{Ca}$ I and $\mathrm{Na} I$ are lower. Histograms for the equivalent line widths of $\mathrm{NaI}, \mathrm{Ca}$ I, and ${ }^{12} \mathrm{CO}(2,0)$, while the water amount for the $15 \mathrm{OH} / \mathrm{IR}$ stars in Schultheis et al. (2003) is also shown in Fig. 7 (dotted line). Their average equivalent widths are: $0.42 \pm 1.56 \AA$ for $E W(\mathrm{Ca}), 2.50 \pm 1.90 \AA$ for $E W(\mathrm{Na})$, and $16.01 \pm 5.16 \AA$ for $E W(\mathrm{CO})$. The equivalent widths for the $\mathrm{OH} / \mathrm{IR}$ stars in this work are: $0.08 \pm 1.88 \AA$ for $E W(\mathrm{Ca})$,
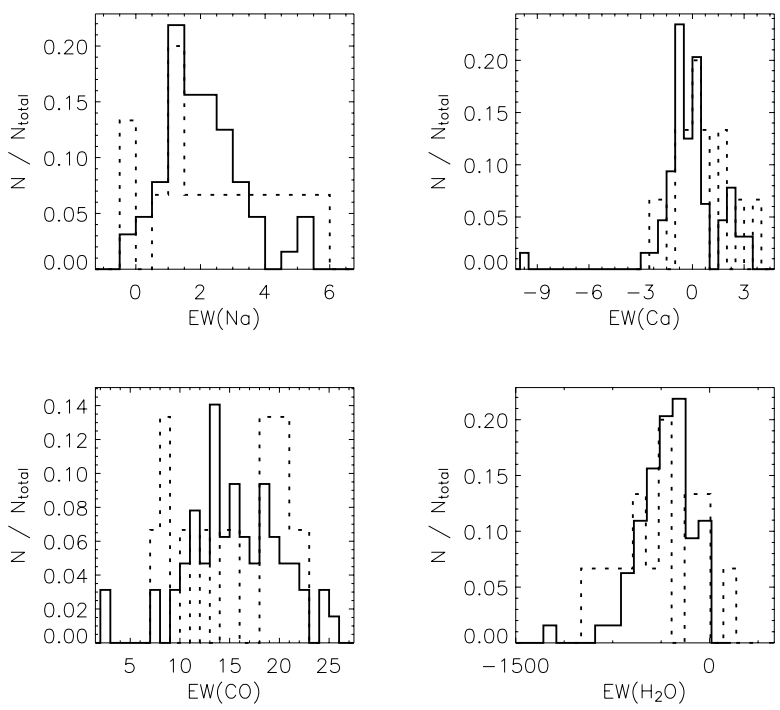

Fig. 7. Histograms for $E W(\mathrm{Ca}), E W(\mathrm{Na}), E W(\mathrm{CO})$ and the water absorption for the $\mathrm{OH} / \mathrm{IR}$ stars from this work (full line) and the OH/IR stars from Schultheis et al. (2003) (dotted line). All $x$-axes are in the same unit $\AA$.

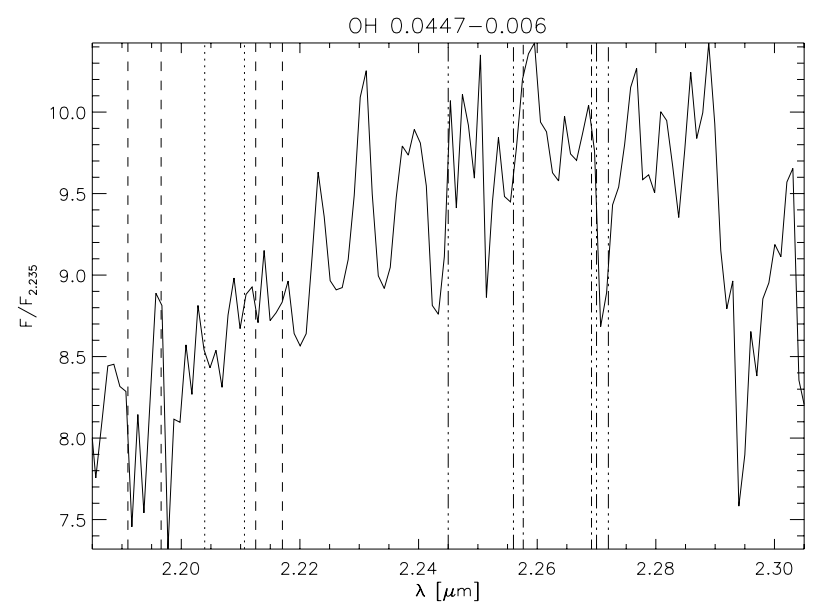

Fig. 8. OH/IR star for which the Na I and Ca I lines could not be measured. The dotted lines indicate the Na I feature and the dashed lines the selected continuum points. The dashed-dotted lines indicate the $\mathrm{Ca}$ I feature with its corresponding continuum (dashed-triple-dotted).

$2.11 \pm 1.27 \AA$ for $E W(\mathrm{Na})$, and $15.60 \pm 4.69 \AA$ for $E W(\mathrm{CO})$ The largest difference is seen for $E W(\mathrm{Ca})$; a lot of the OH/IR stars in this work and in Schultheis et al. (2003) have no measurable Ca I lines.

\section{Discussion}

\subsection{Metallicities of $\mathrm{OH} / \mathrm{IR}$ stars in the $\mathrm{GC}$}

Schultheis et al. (2003) have investigated the metallicity distribution of 107 ISOGAL sources in the GB. The sample consists of different types of stars: non-variable giants, OH/IR stars, supergiants, and LPVs. The metallicity distribution is determined based on the equivalent line widths of $\mathrm{Na}$ I, $\mathrm{Ca}$ I, and ${ }^{12} \mathrm{CO}(2,0)$ (see Ramírez et al. 2000; Frogel et al. 2001; Schultheis et al. 2003), and the calibration of the relation is based on giants in globular clusters in the range $-1.8<[\mathrm{Fe} / \mathrm{H}]<-0.1$. The mean $[\mathrm{Fe} / \mathrm{H}]$ value in Schultheis et al. (2003) is consistent with previous chemical abundance studies of the GB (Schultheis et al. 2003). Apparantly, variable stars, such as Miras and OH/IR stars, 

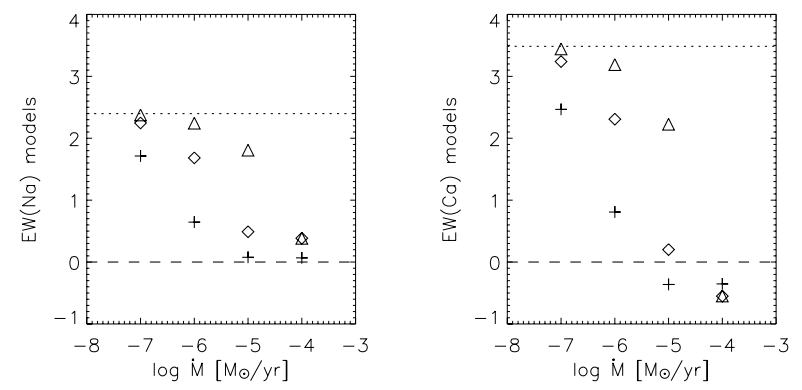

Fig. 9. Equivalent line widths for the different models. The $x$-axis indicates the mass-loss rate, and the $y$-axis the equivalent line widths for $\mathrm{Na}$ I (left) and Ca I (right). The different symbols indicate the different dust temperatures. Crosses: $T_{\text {dust }}=1500 \mathrm{~K}$, diamonds: $T_{\text {dust }}=1000 \mathrm{~K}$ and triangles: $T_{\text {dust }}=750 \mathrm{~K}$. The dotted line indicates the equivalent line width for the reference line. The line disappears when the equivalent width is below the dashed line.

do not influence the peak in the metallicity distribution determined in Schultheis et al. (2003), although they might broaden the distribution. Applying their results to our sample of $\mathrm{OH} / \mathrm{IR}$ stars could help to find a metallicity difference between the two separate groups of $\mathrm{OH} / \mathrm{IR}$ stars in the GC as discussed in the introduction.

The equivalent line widths of $\mathrm{NaI}, \mathrm{CaI}$, and ${ }^{12} \mathrm{CO}(2,0)$ are determined as described in Sect. 3.1. The values in Tables A.1, A.2, and A.3 are given in A. A positive value indicates absorption and a negative value indicates emission. One immediately notices all the negative values for $E W(\mathrm{Ca})$. One does not expect the $\mathrm{CaI}$ and the $\mathrm{NaI}$ lines to be in emission. Inspecting the spectra for these stars (Figs. B.1 to B.4) shows that for a lot of stars the $\mathrm{Ca} I$ lines seem to disappear in the continuum. This effect is also noticeable for $\mathrm{Na}$ I in some stars.

In the rest of this section we discuss the physical effects that can lead to the $\mathrm{Ca}$ I and sometimes the $\mathrm{NaI}$ to appearing in emission.

\subsection{Dust}

$\mathrm{OH} / \mathrm{IR}$ stars are AGB stars in their final phase on the AGB that are believed to be progenitors of Planetary Nebulae (e.g. Cohen et al. 2005; Habing 1996). These stars have high mass-loss rates between $\dot{M}=10^{-6} M_{\odot} /$ yr and $10^{-4} M_{\odot} /$ yr. It is likely that the dust formed around the star has an influence on the near-IR spectrum (Tej et al. 2003).

To study the effect of dust on the Na I and $\mathrm{Ca}$ I lines, the dust radiative transfer model of Groenewegen (1993) was used. In this model, the radiative transfer equation and the radiative equilibrium equation for the dust are solved simultaneously in spherical geometry (Groenewegen et al. 1994). For the dust properties we assume silicate dust (Volk \& Kwok 1988) of radius $a=0.02 \mu \mathrm{m}$ and a specific dust density of $\rho_{\mathrm{d}}=2.0 \mathrm{~g} / \mathrm{cm}^{3}$. As the input model we used a blackbody with $T_{\text {eff }}=2500 \mathrm{~K}$ and with the $\mathrm{Ca}$ I and $\mathrm{Na}$ I lines imposed upon it. A dust-to-gas ratio of $\Psi=0.01$ and an outflow velocity of $v=15 \mathrm{~km} \mathrm{~s}^{-1}$ were assumed. For a grid of mass-loss and dust temperature the models indicate the influence of dust on these lines. Typical AGB massloss rates (between $\dot{M}=10^{-7} M_{\odot} / \mathrm{yr}$ and $10^{-4} M_{\odot} / \mathrm{yr}$ ) and dust temperatures $\left(T_{\text {dust }}=1500,1000\right.$ and $\left.750 \mathrm{~K}\right)$ were used. The model with no mass-loss is used as a reference model.

Figure 9 shows the effect of the increasing dust amount on the equivalent line widths of $\mathrm{NaI}$ and $\mathrm{Ca}$. For both lines a similar trend can be seen in which the equivalent line widths decrease for increasing mass-loss rates. The effect is stronger for higher dust temperatures, except at the $10^{-4} M_{\odot} /$ yr massloss rate where the $\mathrm{NaI}$ and $\mathrm{CaI}$ lines become undetectable for the three dust temperatures. This effect is also stronger with increasing wavelength and affects the $\mathrm{Ca}$ I more than the $\mathrm{Na}$ I. Unfortunately, we do know neither the temperature of the dust, nor the mass-loss rate for our individual sources. We can only demonstrate here the possible influence of the dust on the measured near-infrared spectrum. Surprisingly, negative values for $E W(\mathrm{Ca})$ were measured for these models. This is merely due to the chosen continuum: the change in the continuum's slope is so drastic that it causes negative equivalent line-width measurements.

\subsection{Water content in $\mathrm{OH} / \mathrm{IR}$ stars}

Water absorption lines can influence the near-IR medium resolution spectra of AGB stars severely in a way which depends on the phase of the variable star as shown in Tej et al. (2003). The high-resolution spectrum of $o$ Cet, a M type Mira, in Wallace \& Hinkle (1996) shows that a lot of water lines are situated at wavelengths where the $\mathrm{Ca} I$ and $\mathrm{Na}$ I lines are observed. In the case of the $\mathrm{Ca}$ I line, the strong waterlines are situated where the continuum of the line is determined. The water lines depress the continuum in a medium-resolution spectrum and make the equivalent line-width measurements unreliable. When the depression of the continuum is strong enough, one can expect to start to see the $\mathrm{Ca}$ I line in apparent emission. In the case of the $\mathrm{Na}$ I line, the water lines affect both the continuum and the $\mathrm{Na}$ I line, so that one can expect to see the $E W(\mathrm{Na})$ decrease because of a smaller contrast between the continuum and the line, similar to what is seen due to the influence of the dust on the spectrum, as discussed in the previous subsection.

These effects are reflected in Fig. 10 where the $E W(\mathrm{Ca})$ and the $E W(\mathrm{Na})$ can be seen in comparison with our determination of the water absorption. We see that the RGB stars, with little water absorption, have higher $E W(\mathrm{Ca})$ and the $E W(\mathrm{Na})$ values than the LPVs and OH/IR stars. This effect seems stronger for $\mathrm{Ca}$ I where we basically have no more detections for $E W\left(\mathrm{H}_{2} \mathrm{O}\right)$ below $-300 \AA$. For the Na I line the decrease is more gradual with increasing water absorption. Of course the effect of dust and other factors (such as pulsation amplitude, $T_{\text {eff }}$ ) on the spectra is also included in these figures and cannot be distinguished from the effect that the water absorption has.

\subsection{Variability}

Figure 6 shows the differences in the measured absorption for water clearly for the stars we have in common with Schultheis et al. (2003). The water absorption is expected to be largely correlated with the phase for a given star: the most intense water features are seen at minimum light (Lançon \& Wood 2000; Bessell et al. 1996; Tej et al. 2003).

Wood et al. (1998) determined periods for $80 \mathrm{GC} \mathrm{OH/IR}$ stars. We retrieved $\mathrm{K}$ light curves for 41 out of the 50 stars in common with our sample. The lightcurves were extrapolated using the fundamental period and the first harmonic (Wood 2004) to determine the phase $(=\phi)$ when the star was observed in our campaign. The value of $\phi$ has been calculated in a non-traditional way, such that the maxiumum of the lightcurve is 0 and that the minimum is always at $\phi=0.5$. The values of $0.25 / 0.75$ were fixed when the lightcurve reaches $\langle K\rangle$. The other values for $\phi$ were then interpolated between the fixed ones (see Fig. 11). 

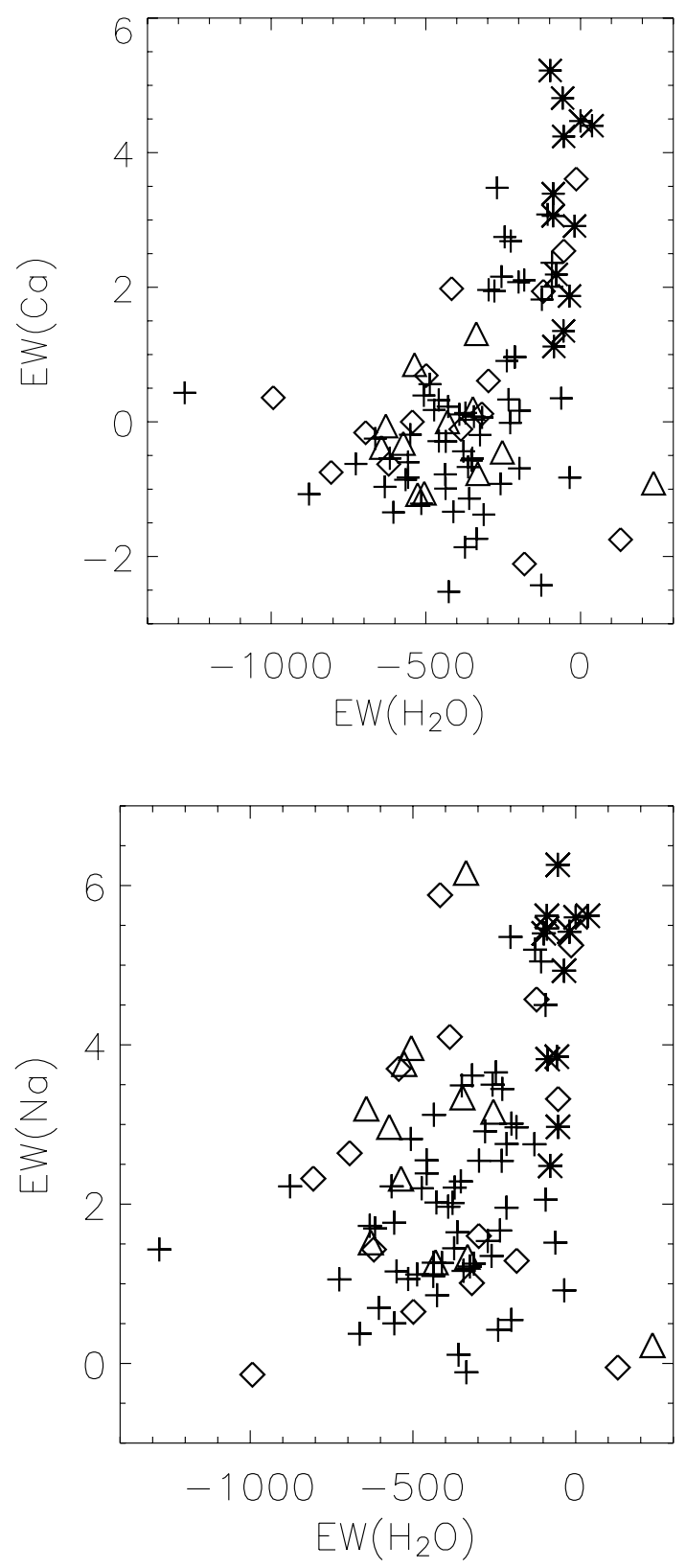

Fig. 10. Upper panel: $E W(\mathrm{Ca})$ vs. $E W\left(\mathrm{H}_{2} \mathrm{O}\right)$, lower panel: $E W(\mathrm{Na})$ vs. $E W\left(\mathrm{H}_{2} \mathrm{O}\right)$. The $\mathrm{OH} / \mathrm{IR}$ in this work are the crosses, RGB (Schultheis et al. 2003): stars, OH/IR (Schultheis et al. 2003): diamonds, LPV (Schultheis et al. 2003): triangles. All equivalent line widths are given in the same unit $\AA$.

Figure 12 shows the phase $\phi$ versus the equivalent line-width of water. The expected correlation can be seen in this figure, but not as clearly as anticipated. Figure 12 indicates that the water absorption is highest for all but one star for $\phi=0.5$, which indicates the light minimum. For the other phases there is a large spread and no clear trend, which is expected as the individual stars have different parameters.

\subsection{Expansion velocities}

Lindqvist et al. (1992b) determined the expansion velocities $v_{\exp }$ for their sample of $\mathrm{OH} / \mathrm{IR}$ stars close to the GC. As mentioned in Sect. 1, they divided the sample into two groups based on the expansion velocities. The first group with the lowest expansion

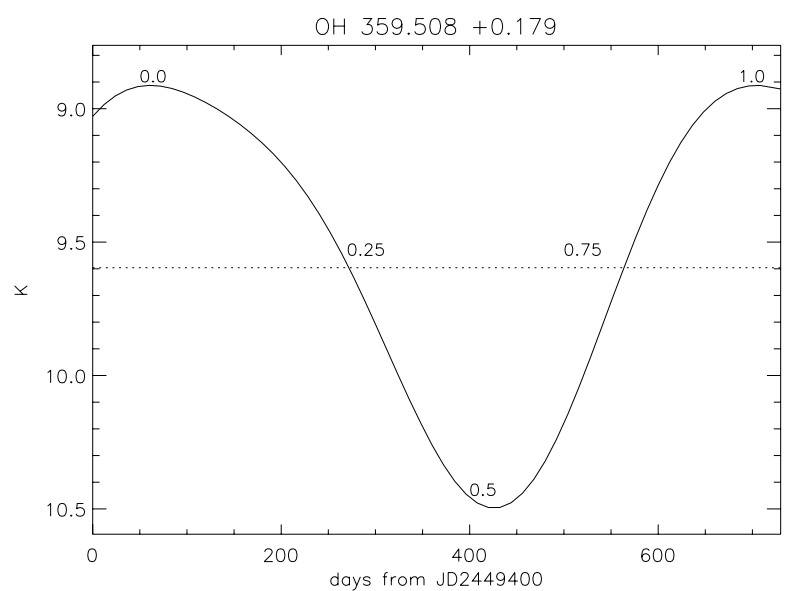

Fig. 11. Determination of the phase $\phi$. The dotted horizontal line gives $\langle K\rangle$.

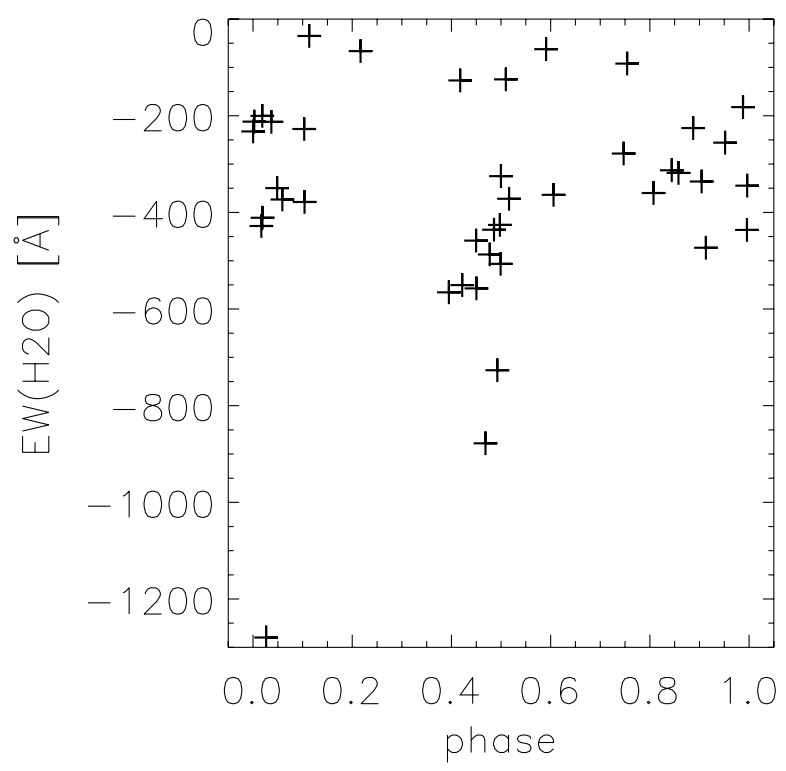

Fig. 12. The phase at time of observation versus the equivalent line width of water absorption.

velocities are kinematically different and show larger dispersions in their radial velocities.

With our spectra we wanted to see whether we could find metallicity differences between the two kinematically different groups. As shown in the previous sections, this is not possible using the relationship based on the equivalent line widths of $\mathrm{Na}$ I, Ca I, and ${ }^{12} \mathrm{CO}(2,0)$ in nonvariable stars with low massloss rates (see Ramírez et al. 2000; Frogel et al. 2001; Schultheis et al. 2003). We still wanted to investigate whether we find differences in the equivalent line widths of the individual lines for the two groups. Figure 13 shows the comparison between the expansion velocities and the equivalent line widths of $\mathrm{Na} \mathrm{I}, \mathrm{Ca}$, ${ }^{12} \mathrm{CO}(2,0)$, and the water absorption around $1.6 \mu \mathrm{m}$, for our sample of $\mathrm{OH} / \mathrm{IR}$ stars. Table 5 gives the mean values with standard deviation for the measured equivalent line widths and the water indication for the both groups. Figure 13 and Table 5 seem to indicate that the $\mathrm{OH} / \mathrm{IR}$ stars in the first group have smaller $E W(\mathrm{Na})$ and a larger spread for the water absorption. Based on the numbers in Table 5, there is no difference between the $E W(\mathrm{CO})$ for both groups. Concerning $\mathrm{Ca}$, the majority of the $\mathrm{OH} / \mathrm{IR}$ stars in the first group have no measurable equivalent 

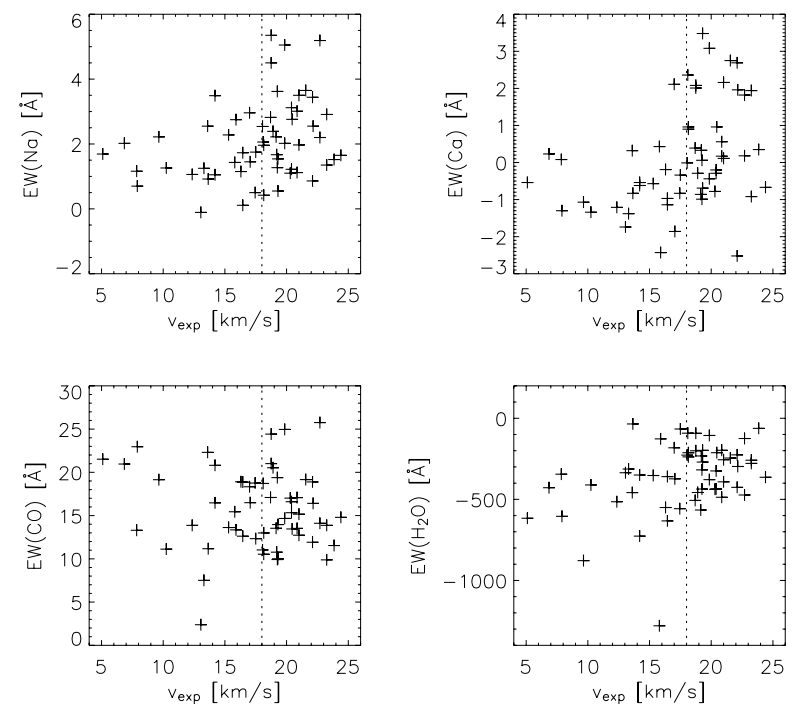

Fig. 13. Comparison of the expansion velocities with the equivalent line widths of Na I, Ca I, ${ }^{12} \mathrm{CO}(2,0)$, and the water absorption. The dotted vertical line separates the 2 groups of $\mathrm{OH} / \mathrm{IR}$ stars.

Table 5. Mean values for the two groups of OH/IR stars.

\begin{tabular}{ccc}
\hline \hline & $v_{\exp }<18 \mathrm{~km} \mathrm{~s}^{-1}$ & $v_{\exp }>18 \mathrm{~km} \mathrm{~s}^{-1}$ \\
\hline $\mathrm{Na}$ I & $1.57 \pm 0.70$ & $2.44 \pm 1.02$ \\
$\mathrm{Ca}$ I & $-0.65 \pm 0.69$ & $0.67 \pm 1.17$ \\
${ }^{12} \mathrm{CO}(2,0)$ & $15.73 \pm 3.90$ & $15.61 \pm 3.44$ \\
$\mathrm{H}_{2} \mathrm{O}$ & $-452.90 \pm 190.82$ & $-294.97 \pm 110.31$ \\
\hline
\end{tabular}

line widths. Since the water absorption has a larger spread for this group and because of the dependence seen in Fig. 10, it is consistent that we measure negative $E W(\mathrm{Ca})$ for the majority of these stars.

Decin (2000) indicates that the linestrength of $\mathrm{H}_{2} \mathrm{O}$ increases with decreasing metallicity. This could explain why we see stronger water absorption for the $\mathrm{OH} / \mathrm{IR}$ stars in the first group, provided these $\mathrm{OH} / \mathrm{IR}$ stars are older and have lower metallicities than the stars in the second group, as suggested by kinematics and outflow velocities.

\section{Conclusions}

We obtained near-IR data $(1.53-2.52 \mu \mathrm{m})$ with SOFI on the NTT for $70 \mathrm{OH} / \mathrm{IR}$ stars located in the GC. The spectra were analysed based on the equivalent line widths of $\mathrm{Na}$ I, Ca I, and ${ }^{12} \mathrm{CO}(2,0)$. The curvature of the spectrum around $1.6 \mu \mathrm{m}$ indicates the amount of water. The equivalent line widths of $\mathrm{NaI}$, $\mathrm{Ca}$, and ${ }^{12} \mathrm{CO}(2,0)$ were found to have low values in comparison to the GC static giant stars. For a large fraction of the OH/IR stars, we even found that $\mathrm{Ca}$ I lines could not be detected.

We discuss different elements which can influence the determination of the equivalent line widths in the near-infrared spectra. The OH/IR stars have a variable amount of water, which especially influences the Ca I lines. The water lines just beside the $\mathrm{Ca}$ I line depress the continuum, causing the $\mathrm{Ca}$ I lines to disappear in the spectrum. The effect is also noticeable for $\mathrm{Na}$ I but is not as strong.
We also discuss the effect of the circumstellar dust on the near-infrared spectrum. By using the radiative transfer model (Groenewegen 1993), it became clear that for the highest massloss rates the dust has the same effect on the $\mathrm{Ca}$ I and $\mathrm{Na} I$ lines as the water content: the lines become weaker and in the extreme case of mass-loss rates of the order of $10^{-4} M_{\odot} /$ yr even disappear in the continuum and are no longer measurable. For the lower mass-loss rates $\left(\sim 10^{-6} M_{\odot} / y r\right)$, the decrease in the equivalent line widths depends strongly on the dust temperature. Since we do not know the exact dust temperature and the mass-loss rates of these $\mathrm{OH} / \mathrm{IR}$ stars, we cannot distinguish between the possible effects that weaken the lines. The different effects discussed above prevent us from finding a clear distinction between the two groups of OH/IR stars.

Acknowledgements. M.S. is supported by an APART fellowship. We want to thank P.R. Wood, who kindly provided us with the K lightcurves for the OH/IR stars.

\section{References}

Aringer, B., Jorgensen, U. G., \& Langhoff, S. R. 1997, A\&A, 323, 202

Baud, B., Habing, H. J., Matthews, H. E., \& Winnberg, A. 1981, A\&A, 95, 156 Bessell, M. S., Scholz, M., \& Wood, P. R. 1996, A\&A, 307, 481

Blommaert, J. A. D. L., van der Veen, W. E. C. J., \& Habing, H. J. 1993, A\&A, 267, 39

Blommaert, J. A. D. L., van der Veen, W. E. C. J., van Langevelde, H. J., Habing, H. J., \& Sjouwerman, L. O. 1998, A\&A, 329, 991

Cardelli, J. A., Clayton, G. C., \& Mathis, J. S. 1989, ApJ, 345, 245

Cohen, M., Parker, Q. A., \& Chapman, J. 2005, MNRAS, 357, 1189

Decin, L. 2000, Ph.D. Thesis

Elitzur, M., Ivezić, Ž., \& Vinković, D. 2003, in Mass-Losing Pulsating Stars and their Circumstellar Matter, ASSL, 283, 265

Frogel, J. A., Stephens, A., Ramírez, S., \& DePoy, D. L. 2001, AJ, 122, 1896

Groenewegen, M. A. T. 1993, Ph.D. Thesis

Groenewegen, M. A. T., de Jong, T., \& Gaballe, T. R. 1994, A\&A, 287, 163

Habing, H. J. 1996, A\&AR, 7, 97

Habing, H., \& Whitelock, P. 2003, in Asymptotic giant branch stars, by H. J. Habing \& H. Olofsson, Astronomy and astrophysics library (New York, Berlin: Springer), 411

Habing, H. J., Tignon, J., \& Tielens, A. G. G. M. 1994, A\&A, 286, 523

Jones, T. J., McGregor, P. J., Gehrz, R. D., \& Lawrence, G. F. 1994, AJ, 107, 1111

Lançon, A., \& Wood, P. R. 2000, A\&AS, 146, 217

Lindqvist, M., Habing, H. J., \& Winnberg, A. 1992a, A\&A, 259, 118

Lindqvist, M., Winnberg, A., Habing, H. J., \& Matthews, H. E. 1992b, A\&AS, 92,43

Omont, A., Gilmore, G. F., Alard, C., et al. 2003, A\&A, 403, 975

Origlia, L., Moorwood, A. F. M., \& Oliva, E. 1993, A\&A, 280, 536

Ortiz, R., Blommaert, J. A. D. L., Copet, E., et al. 2002, A\&A, 388, 279

Ramírez, S. V., Depoy, D. L., Frogel, J. A., Sellgren, K., \& Blum, R. D. 1997, AJ, 113, 1411

Ramírez, S. V., Stephens, A. W., Frogel, J. A., \& DePoy, D. L. 2000, AJ, 120, 833

Schultheis, M., Ganesh, S., Simon, G., et al. 1999, A\&A, 349, L69

Schultheis, M., Lançon, A., Omont, A., Schuller, F., \& Ojha, D. K. 2003, A\&A, 405,531

Sevenster, M. N., Chapman, J. M., Habing, H. J., Killeen, N. E. B., \& Lindqvist, M. 1997, A\&AS, 122, 79

Sjouwerman, L. O., van Langevelde, H. J., Winnberg, A., \& Habing, H. J. 1998, A\&AS, 128, 35

Tej, A., Lançon, A., Scholz, M., \& Wood, P. R. 2003, A\&A, 412, 481

van Langevelde, H. J., Brown, A. G. A., Lindqvist, M., Habing, H. J., \& de Zeeuw, P. T. 1992, A\&A, 261, L17

Volk, K., \& Kwok, S. 1988, ApJ, 331, 435

Wallace, L., \& Hinkle, K. 1996, ApJS, 107, 312

Wood, P. R. 2004, private communication

Wood, P. R., Whiteoak, J. B., Hughes, S. M. G., et al. 1992, ApJ, 397, 552

Wood, P. R., Habing, H. J., \& McGregor, P. J. 1998, A\&A, 336, 925 